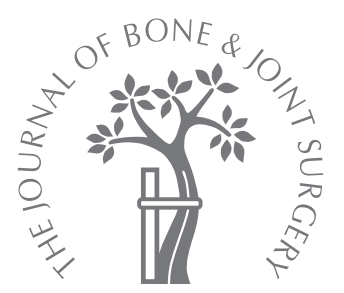

R. J. Gillespie,

A. Levine,

S. J. Fitzgerald,

J. Kolaczko,

M. DeMaio,

R. E. Marcus,

D. R. Cooperman

From University

Hospitals Case

Medical Centre,

Ohio, United States

R. J. Gillespie, MD,

Orthopaedic Surgeon

A. Levine, MD, Orthopaedic

Surgery Resident

S. J. Fitzgerald, MD

Orthopaedic Surgeon

J. Kolaczko, Undergraduate

Student

R. E. Marcus, MD, FACS,

Orthopaedic Surgeon,

Professor and Chairman

D. R. Cooperman, MD

Orthopaedic Surgeon,

Professor

Department of Orthopaedic

Surgery

Case Western Reserve

University, 11100 Euclid

Avenue, Cleveland, Ohio 44106

USA.

M. DeMaio, MD, Orthopaedic Surgeon, Associate Professor

Department of Orthopaedic

Surgery

Naval Medical Center, 620 John

Paul Jones Circle, Portsmouth,

Virginia 23708, USA.

Correspondence should be sent to Dr R. J. Gillespie; e-mail:

robertgillespie77@gmail.com

(C)2011 British Editorial Society of Bone and Joint Surgery doi:10.1302/0301-620X.93B3. $24708 \$ 2.00$

$J$ Bone Joint Surg $[\mathrm{Br}]$ 2011;93-B:357-63.

Received 18 February 2010;

Accepted after revision 26

October 2010

\title{
Gender differences in the anatomy of the distal femur
}

\begin{abstract}
Recently, gender-specific designs of total knee replacement have been developed to accommodate anatomical differences between males and females. We examined a group of male and female distal femora matched for age and height, to determine if there was a difference in the aspect ratio (mediolateral distance versus anteroposterior distance) and the height of the anterior flange between the genders. The Hamann-Todd Collection provided 1207 skeletally mature cadaver femora. The femoral length, the anteroposterior height, height of the lateral and medial flanges and the mediolateral width were measured in all the specimens. The mechanical axis of the femur, the cut articular width and the aspect ratio were assessed. Statistical analysis of the effect of gender upon the aspect ratio and the lateral and medial flanges was undertaken, controlling for age, height and race.

The mean aspect ratio of male femora was 1.21 (SD 0.07 ) and of female femora it was 1.16 (SD 0.06) $(p<0.001)$. There was no significant difference between male and female specimens in the mean size of the lateral flange $(6.57 \mathrm{~mm}$ (SD 2.57) and $7.02 \mathrm{~mm}$ (SD 2.36), respectively; $p=0.099$ ) or of the medial flange $(3.03 \mathrm{~mm}$ (SD 2.47) and $3.56 \mathrm{~mm}$ (SD 2.32), respectively; $p=0.67$ ). Future work in the design of knee prostheses should take into account the overall variability of the anatomy of the distal femur.
\end{abstract}

The number of designs and sizes available for total knee replacement (TKR) in the United States has increased dramatically since the introduction of the first hinged design in the 1950s. It is imperative for the components to be of the appropriate size. If the femoral component is too large there is a risk of overstuffing the joint, thus limiting movement and if it is smaller than the native knee, there is a risk of notching of the femur or over-resection of the posterior condyles, which may lead to instability in flexion.

Recent studies have shown that women have a similar functional outcome to men ${ }^{1-3}$ but increased pain ${ }^{4}$ following TKR. None identified any factors to account for these differences and it remains unclear if they are due to the design of the implant or other factors.

Gender-specific designs of TKR have been developed in an effort to accommodate anatomical differences of the distal femur between men and women. ${ }^{5,6}$ These designs incorporated a smaller aspect ratio, namely, the ratio of the mediolateral (ML) distance to the anteroposterior (AP) distance, as well as a reduced anterior flange and an increased angle of the trochlear groove for the female knee. It is unclear whether the new designs will improve the clinical outcome in women. ${ }^{7}$ Many studies have addressed the proposed difference between genders. ${ }^{1,8-11}$ Some have shown that there is a difference whereas others have not. However, none have strictly controlled for variables such as age, height, length of the femur or race.

The aim of this study, therefore, was to examine an age- and height-matched group of male and female distal femora to determine whether there is a gender difference in the aspect ratio (ML/AP) or the height of the anterior flange.

\section{Materials and Methods}

Femora were obtained from the Hamann-Todd human osteological collection at the Cleveland Museum of Natural History. This contains complete disarticulated human skeletons gathered from unclaimed remains at the Cleveland city morgue between 1912 and 1938. All female specimens older than 18 years with a recorded age and race were included. Exclusion criteria were evidence of a fracture (20), excessive hypoplasia (nine), arthritic changes to the distal femur which would prevent measurements (58) and degradation or post-mortem damage (121) which would prevent accurate point capture and measurements. Using grouped-match analysis, male specimens older than 18 years 
Table I. Clinical details of the cadaver specimens

\begin{tabular}{|c|c|c|c|}
\hline & Female $(n=660)$ & Male $(n=547)$ & Total $(n=1207)$ \\
\hline Mean age (SD) in yrs & $43.3(16.2)$ & $44.3(15.7)$ & $43.7(16.0)$ \\
\hline Mean height (SD) in $\mathrm{cm}$ & $1629.4(64.1)$ & $1652.0(75.2)$ & $1639.6(70.3)$ \\
\hline Mean femoral length (SD) in $\mathrm{mm}$ & $428.6(22.1)$ & $441.5(27.9)$ & $434.4(25.7)$ \\
\hline African-American (\%) & $406 \quad(61.5)$ & $118 \quad(21.6)$ & $524 \quad(43.4)$ \\
\hline
\end{tabular}

with a known race and age were also selected from the collection to match the measured female femora by the height and age of the specimens (Table I). The same exclusion criteria for the female specimens were applied to the male femora. Some male and female specimens had only one side included in the study.

Design of the study. The study had two components. First, the inter- and intra-observer reliability of our method of data collection was assessed. Ten specimens were selected randomly and four of the authors (AL, JK, SJF, RJG) measured each femur twice and the reliability of measurements and calculations for the femur were assessed.

The gender differences between the aspect ratio and the height of the lateral and medial flanges were then assessed. All 513 female specimens in the collection were inspected, and 355 met the inclusion criteria. A total of 660 female femora were measured as not all the specimens had two femora which met the inclusion criteria. Using groupedmatch analysis, 303 male specimens (547 femora) were matched to the female femora, controlling for age, height and race (Table $\mathrm{I}$ ).

Femoral measurements. We used the Microscribe G2LX digitiser (Immersion Corporation, Columbia, Maryland), Microsoft Excel (Microsoft Corporation, Redmond, Washington) and MATLAB software (The Mathworks, Natick, Massachusetts) for collection and analysis of data. The Microscribe digitiser was used to capture points on each femur by its mechanical arm with a stylus which could digitise three-dimensional points from any surface with an accuracy of up to $0.22 \mathrm{~mm}$. MATLAB software provided a high level of programming which allowed for development of an algorithm analysis and visualisation of data and numerical computation. New MATLAB software routines were written to perform geometrical analyses of the digitised morphology of the femur and to create a Microsoft Excel spreadsheet of measurements using a threedimensional Cartesian co-ordinate grid.

After we had secured the digitiser and the femur to a flat surface, data points were obtained in a sequential fashion. These included the femoral head, the distal anterior femoral metaphysis on the lateral side, the anteromedial flange, the anterolateral flange, the medial epicondyle, the lateral epicondyle, the most proximal aspect of the trochlear groove, the distal femoral condyle on the lateral and medial side and the most lateral and medial aspects of the articular surface. The posterior femoral condyles were used as the reference point for measurements of AP height.
The length of the femur, the mechanical axis, the height of the lateral and medial flanges, the height of the standard anterior cut and the width of the articular surface were calculated (Fig. 1).

Using MATLAB software and the digitiser, a distal femoral resection was generated for each specimen. We simulated a resection of $8 \mathrm{~mm}$ from the most distal femoral condyle, perpendicular to the mechanical axis of the femur. Points along the lateral and medial aspect of the distal femur at the level of the epicondylar axis determined the width of the femur after the simulated resection. Since most TKR systems require an initial cut of between $7 \mathrm{~mm}$ to $10 \mathrm{~mm}$ from the distal femur, $8 \mathrm{~mm}$ was chosen. The aspect ratio was calculated from these measurements (Fig. 1).

Statistical analysis. Inter- and intra-observer reliability was assessed using the variance components of a mixed-effects model which accounted for the correlation between and within raters based on the initial measurements of the ten femora.

For the analysis of variables, summary measures were recorded as the mean (SD) for continuous variables (femoral length, height, age) and as a percentage count for dichotomous variables (gender, race). Demographic variables such as age, height and race were specimen level variables, whereas femoral length was a femoral level variable. No difference was found between the summary statistics calculated at the specimen level versus those at the femoral level. Table I was calculated at the femoral level. The descriptive summary statistics in Table II were also calculated at the femoral level.

The effects of interest reported in Table II were calculated in a mixed-effects modelling framework which accounted for the correlation between femora taken from the same specimen. Covariates for the models were gender, femoral length and race. Age was considered to be a covariate, but was not found to be significant in any setting.

Correlation and concordance coefficients were calculated to compare the agreement between left and right femora from the same specimen. Analysis of the overlap of gender distributions was based on assuming a normal distribution of the values of the aspect ratio, using the mean (SD) for females (Table II) and the estimated effect size from the model (Table II). All the statistical analyses were performed using R (R Foundation for Statistical Computing, Vienna, Austria). A p-value $\leq 0.05$ was considered to be significant. 


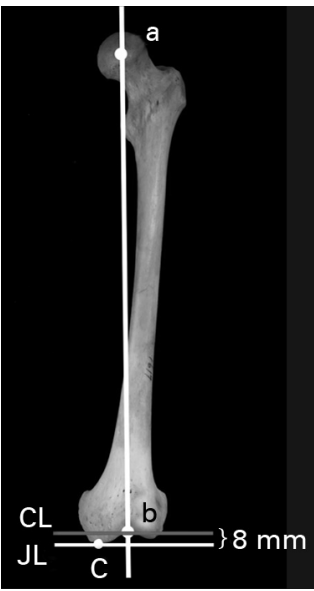

Fig. 1a

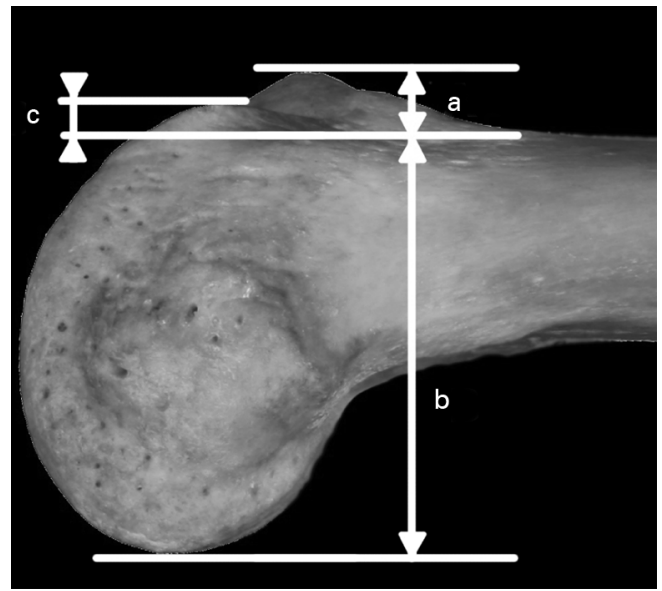

Fig. 1b

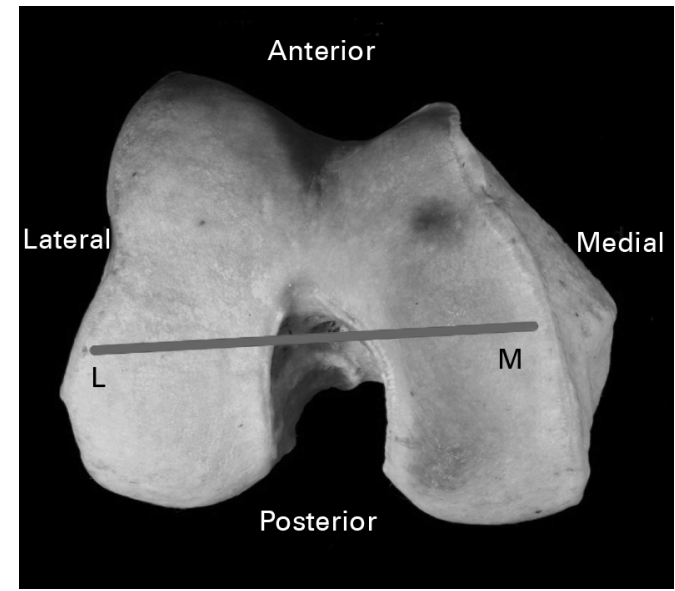

Fig. 1c

Photographs showing a) an anteroposterior view of a femur ( $a$, centre of the femoral head; $b$, centre of the trochlear groove; $a-b$, the mechanical axis of the femur; c, the most distal point of the distal femur; JL, the joint line; and CL, the simulated distal femoral cut line 8 mm proximal to the joint line), b) a lateral view of a distal femur ( $a+b$, the overall anteroposterior height; $a$, the lateral flange height; $b$, the minimal anterior cut height; and $\mathrm{c}$, the medial flange height), and c) an axial view of the distal femur (M-L, mediolateral length used to calculate the aspect ratio).

Table II. Measurements of the distal femur. A positive gender effect indicates a larger value in males. The femoral length effect is taken for the entire population. A positive femoral length effect indicates for an increasing length of the femur, the variable being studied also increases. A positive race effect indicates a larger value in the African-American population

\begin{tabular}{|c|c|c|c|c|c|c|c|c|}
\hline & Female (660) & Male (547) & $\begin{array}{l}\text { Gender } \\
\text { effect }\end{array}$ & p-value & $\begin{array}{l}\text { Femoral length } \\
\text { effect }\end{array}$ & p-value & Race effect & p-value \\
\hline Aspect ratio (SD) & $1.16(0.06)$ & $1.21(0.07)$ & 0.06 & $<0.001$ & -0.0002 & 0.041 & 0.02 & $<0.001$ \\
\hline Mean (SD) lateral flange height in mm & $7.02(2.36)$ & $6.57(2.57)$ & 0.28 & 0.099 & 0.033 & $<0.001$ & 2.6 & $<0.001$ \\
\hline Mean (SD) medial flange height in $\mathrm{mm}$ & $3.56(2.32)$ & $3.03(2.47)$ & 0.019 & 0.917 & 0.018 & $<0.001$ & 2.0 & $<0.001$ \\
\hline
\end{tabular}

\section{Results}

Reliability measurements. The amount of variability associated with the four different investigators was an order of magnitude smaller than that associated with different femora. Also, the investigator and replication components had similar size variability, indicating that the effect of different investigators was similar to the effect of repeated measurements by the same investigator.

Aspect ratio. Male and female femora were similar with respect to age and height (group-matched) and the femoral length correlated with height (Table I). The mean aspect ratio of male specimens was 1.21 (SD 0.07) and for females, 1.16 (SD 0.06) (Table II). There was a positive male gender effect of $0.06(\mathrm{p}<0.001)$ after adjusting for all other covariables, including race (Fig. 2a). There was a large amount of variability between male and female specimens relative to the size of the gender effect, leading to a high amount of overlap between genders. African-American specimens had a larger aspect ratio by a mean of 0.02 compared with Caucasian femora ( $<<0.001$; Fig. 2b). There was a mean decrease of 0.0002 in the aspect ratio for an increase of $1 \mathrm{~mm}$ in the femoral length for the entire study population ( $\mathrm{p}=0.041$; Fig. 3 ). The correlation and concordance between left and right femora from the same skeleton were high (0.82).
Height of the lateral flange. A similar analysis was performed for the lateral flange of the distal femur. Its mean height for the male specimens was $6.57 \mathrm{~mm}$ (SD 2.57) and for the female femora it was $7.02 \mathrm{~mm}$ (SD 2.36) (Fig. 4a). After adjusting for all covariates, there was no significant effect of gender on the size of the lateral flange ( $p=0.099$; Table II). Any supposed effect of gender on its height could be accounted for by the effect of race between African-American and Caucasian specimens. African-American male and female specimens had an increased effect (2.6) on the height of the lateral flange compared with the Caucasian femora ( $p<0.001$; Fig. 4b). Similar to the aspect ratio, the height of the lateral flange was related to the length of the femur for the entire study population, with a mean increase in height of $0.03 \mathrm{~mm}$ for every increase of $1 \mathrm{~mm}$ in the femoral length $(\mathrm{p}<0.001)$. The concordance $(0.89)$ and correlation $(0.89)$ between the left and right femora from the same skeleton were high.

Height of the medial flange. A similar analysis was performed for the media flange of the distal femur (Table II). The mean height of the medial flange of the male specimens was $3.03 \mathrm{~mm}$ (SD 2.47) and of the female specimens it was $3.56 \mathrm{~mm}$ (SD 2.32) (Fig. 5a). After adjusting for covariates, there was no significant effect of gender on the size of the 


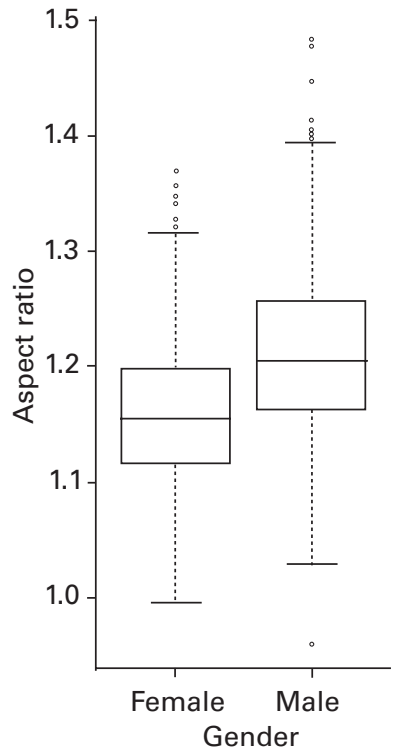

Fig. 2a

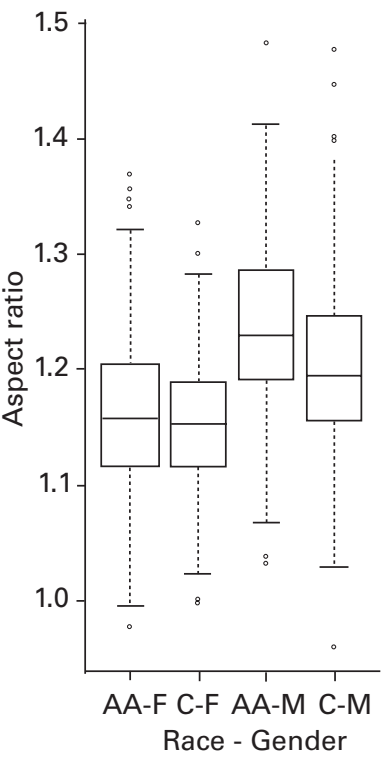

Fig. $2 b$
Box-plots showing the aspect ratio versus a) gender and b) race and gender (AA, African-American; C, Caucasian; F, female; M, male).

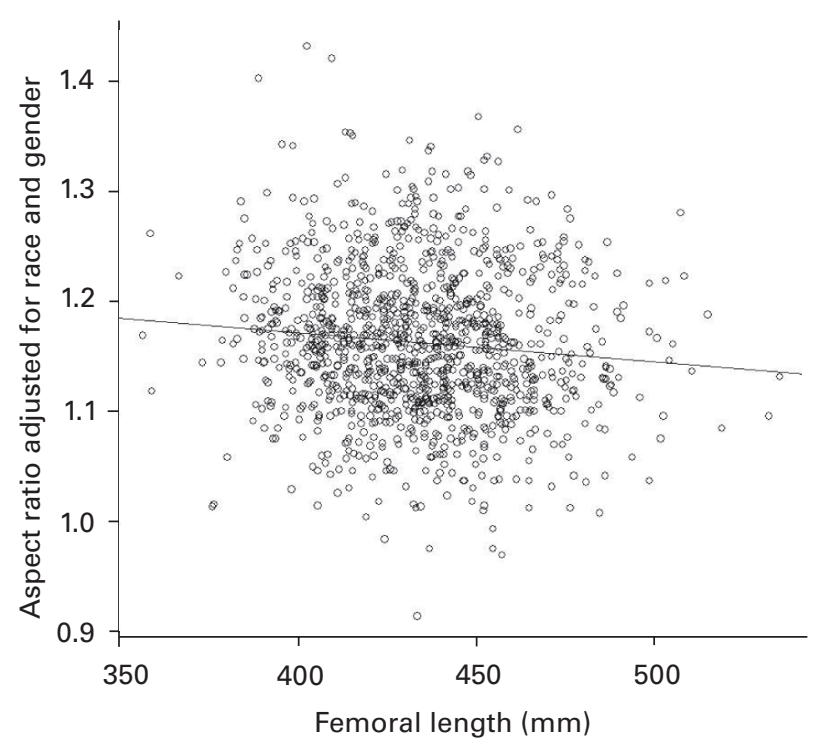

Fig. 3

Scattergram showing the effect of femoral length on the race- and gender-adjusted aspect ratio.

medial flange $(\mathrm{p}=0.67)$. The male and female distribution of the height of the medial flange almost completely overlapped. Similar to the lateral flange, a positive effect (2.0) was found between African-American and Caucasian specimens ( $\mathrm{p}<0.001$; Fig. 5b). The height of the medial flange

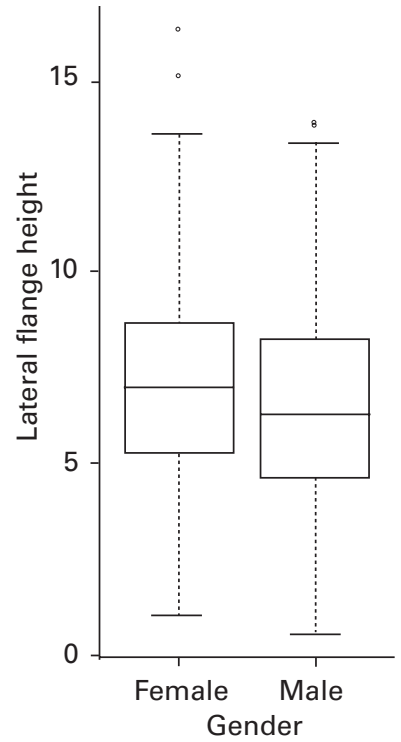

Fig. $4 a$

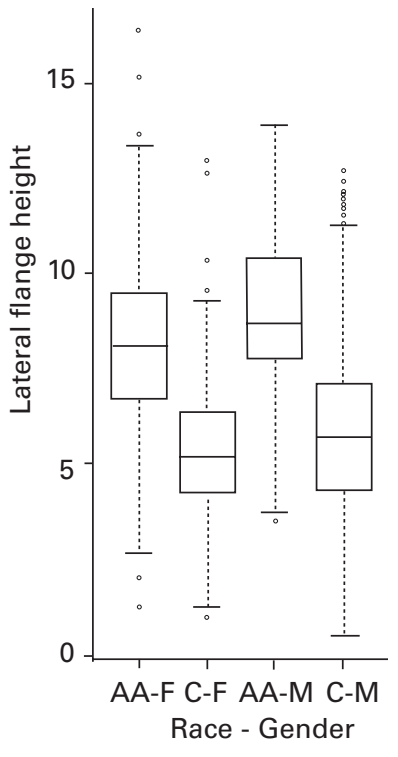

Fig. 4b
Box-plots showing the height of the lateral flange $(\mathrm{mm})$ versus a) gender and b) race and gender (AA, African-American; C, Caucasian; F, female; $M$, male).

was related to the length of the femur, with a mean increase of $0.02 \mathrm{~mm}$ in height for every increase of $1 \mathrm{~mm}$ in femoral length for the entire study population $(\mathrm{p}<0.001)$. The concordance $(0.85)$ and correlation (0.86) between left and right femora from the same skeleton were high.

\section{Discussion}

As the design of systems for TKR continues to evolve, it is imperative that anatomical differences between genders be taken into account. The specimens used in our study were collected in the early 20th century. The mean age of this population was 44 years (SD 16) and a large proportion would have been in the generation of patients who would be candidates for a TKR in the modern orthopaedic era. This allows for some conclusions to be drawn regarding anatomical differences between gender and race in the anatomy of the distal femur as it relates to the modern knee prostheses. In our study, there was a significant difference in the aspect ratio between the male and female distal femora $(p<0.001)$, but we found that there was a high degree of variability between individuals. However, there was no difference in the height of the lateral and medial flanges between male and female specimens ( $\mathrm{p}=0.099$ and 0.67 ; respectively).

Osteology is often used to assign gender and age in forensic medicine and paleontology. The distal femur is not an area routinely or solely used to determine gender. ${ }^{12}$ Recently, Mahfouz et $\mathrm{al}^{5}$ described many differences in the anatomy of the distal femur between men and women. However, their study had limitations including a small number of specimens and the use of computer-assisted 


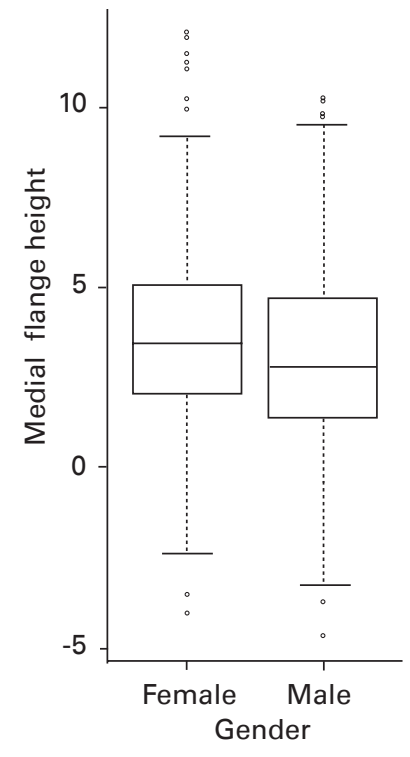

Fig. $5 a$

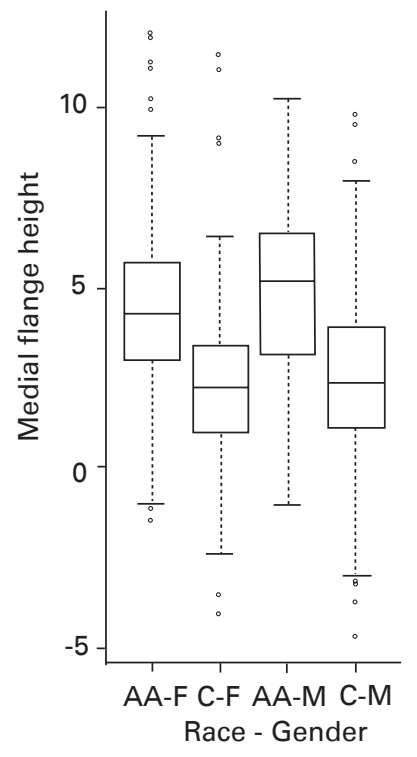

Fig. $5 b$
Box-plots showing the height of the medial flange $(\mathrm{mm})$ versus a) gender and $b$ ) race and gender (AA, African-American; C, Caucasian; F, female; $M$, male).

enlargements to compare femora which assumes that long and short bones are symmetrical. Lonner et $\mathrm{al}^{1}$ showed that there were differences and variability between and within genders, suggesting that the shapes of the distal femora of men and women did not resemble one another when size overlapped. Hitt et $\mathrm{al}^{8}$ also found that when compared with standard available implants, overhang was significantly greater in female specimens. Both of these studies used intra-operative measurements on a relatively small sample size without controlling for the height or length of the femur. Bellemans et $\mathrm{al}^{13}$ also studied the effect of gender on the anatomy of the distal femur and found that both gender and morphotype influenced its shape. Recently, Fehring et $\mathrm{al}^{10}$ examined the heights of the lateral and medial flanges of men and women from MRI studies and found a small difference in the height of the medial flange, but not of the lateral flange. Despite these small differences, they found a large amount of variability within the groups emphasising the need for the design of TKR to be based on the overall variability of the human anatomy, and not necessarily on gender.

We found that African-American specimens had a larger aspect ratio and larger heights of the lateral and medial flanges compared with the Caucasian femora after adjusting for femoral length and gender. Other studies have also examined race as a cause of anatomical differences in the distal femur. Vaidya et $\mathrm{al}^{14}$ found that most Indian males could have a satisfactory replacement with the currently available knee designs while significantly fewer Indian females could be suitably accommodated. However, their study examined the limitations of the AP dimension more

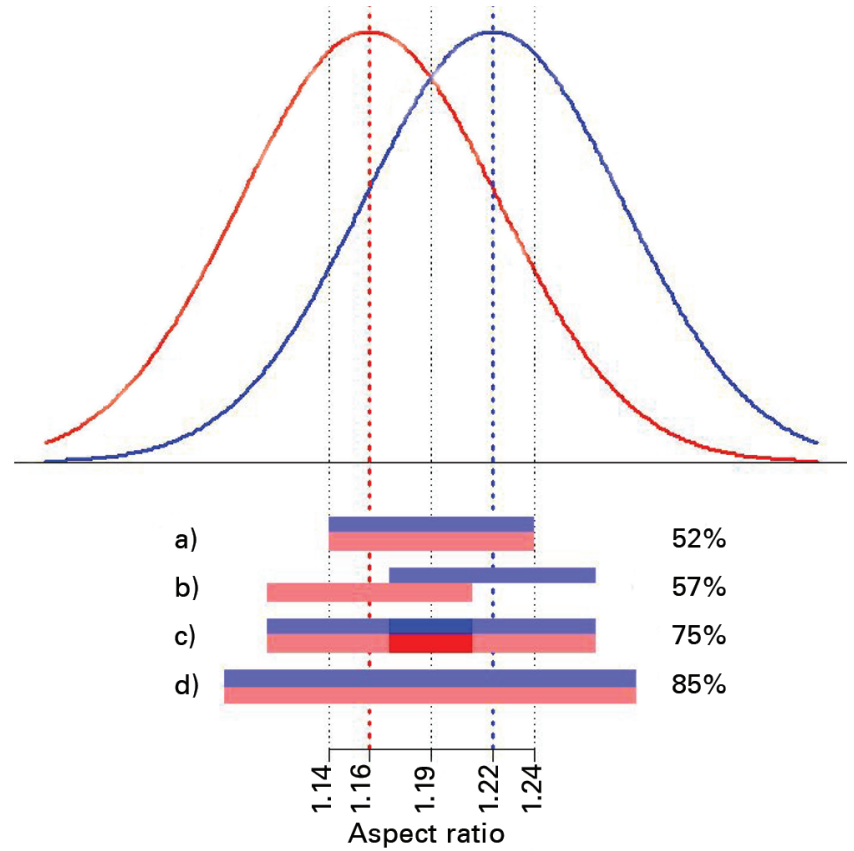

Fig. 6

Normal curves showing the distribution of male (blue) and female (red) aspect ratios. The bars in a) to d) indicate the percentage of patients achieving an ideal-fit prosthesis in the centre of a) one design with the aspect ratio at the overall mean $(1.19)$; b) two designs with aspect ratios at the mean of males (1.22) and females (1.16), respectively; $c$ ) the same designs as $b$, but both designs available to either gender; d) two designs with aspect ratios \pm 0.05 (range 1.14 to 1.24 ) of the overall mean, available to either gender.

Table III. Mean (range) aspect ratio, for a range of femoral components from four implant companies

\begin{tabular}{ll}
\hline Implant $^{*}$ & Mean aspect ratio (range) \\
\hline Stryker Triathlon & $1.09(1.06$ to 1.11$)$ \\
DePuy Sigma & $1.07(1.05$ to 1.09$)$ \\
Smith \& Nephew Journey & $1.11(1.04$ to 1.15$)$ \\
Zimmer NexGen LPS & $1.11(1.06$ to 1.18$)$ \\
Zimmer NexGen Flex & $1.10(1.06$ to 1.16$)$ \\
Zimmer NexGen Gender & 1.05 (1.01 to 1.09$)$ \\
* Stryker (Kalamazoo, Michigan), DePuy (Warsaw, Indiana), \\
Smith \& Nephew (Memphis, Tennessee), Zimmer (Warsaw, \\
Indiana)
\end{tabular}

than the aspect ratio. Cheng et $\mathrm{al}^{15}$ examined CT data of a Chinese population, showing that males had a higher aspect ratio compared with females. Based on our study and the recent literature, race contributes to the variability in the size and shape of the distal femur, but no conclusions can be made regarding the effect of these differences on a TKR.

The information from our study has potential ramifications in the design of TKRs since some studies on clinical outcome have identified differences in males and females. ${ }^{2-4}$ 
No difference was found in the height of the flanges between genders and the aspect ratio had considerable overlap and variability, with female specimens tending to have a smaller aspect ratio than male femora of any given length.

The question remains as to the role that gender plays in the design of future TKRs. Although this is a cadaver study in which no definitive conclusions on clinical outcome can be made, the role that overall variability, and not necessarily gender differences, has in the design of TKRs can be illustrated. An 'ideal fit' of the femoral component occurs when excessive overhang and overstuffing of the knee is avoided. If an ideal fit of a TKR is defined as an aspect ratio within 0.05 of the implant aspect ratio, and there was only one implant system with an aspect ratio based on the mean of this population (1.19), $52 \%$ of the population would have a femoral component which was an ideal fit. Alternatively, if an implant company had two designs for the distal femur, one based on the aspect ratio of women (1.16) and the other based on that of men (1.22), after considering the gender effect $(0.06)$ in the population $57 \%$ would have an ideal fit if all males received the 'male knee' and all females the 'female knee'. However, if males could receive either the 'female' or 'male' implant and females could receive either the 'male' or 'female' implant, the percentage of patients in this population who would receive an ideal-fit prosthesis would increase to $75 \%$. Finally, if the two designs were based on an aspect ratio which was \pm 0.05 from the mean of this population $(1.19 ; 1.14$ to 1.24$)$, the percentage of patients who would receive an ideal-fit prosthesis would increase to $85 \%$ (Fig. 6). This indicates that the design of future TKRs should be based on the overall variability in the shape of the distal femur in the population and not on gender differences. At this time, although there is some variability of the aspect ratio between designs of the knee (Table III), it is clear from this and other studies that there is more variation in the aspect ratio of the human population than in that of implants. ${ }^{8}$ In addition, the overall variability in the aspect ratio for each implant system is not high, possibly limiting the number of patients who would achieve an ideal fit when only one implant system is available. Future studies should therefore focus on the fit of an implant as it relates to the patient's clinical outcome in order to design a prosthetic system with enough variability in terms of aspect ratio for most patients to receive an ideal fit.

A major strength of our study is the large sample size, giving it the power to make significant conclusions regarding aspect ratio and flange height, while accounting for other variables such as race, age and height. In addition, the digitised femoral measurements were reliable and accurate. However, there were several limitations. This was a cadaver study of specimens obtained in the beginning of the 20th century. Although many would have been candidates for a TKR in the modern era if they had survived, there was no control of the environmental or nutritional factors which may have limited the significance of the results seen in this population. However, the mean height of Caucasian and
African-American females in the United States today is approximately $163 \mathrm{~cm},{ }^{16}$ which was the mean height of the females in our study population. Our study matched males who were the same height as the females to control for height as a variable, and therefore no conclusions can be drawn regarding very tall individuals (Table I). We assume that our observation that the aspect ratio decreases by 0.0002 for every increase of $1 \mathrm{~mm}$ in femoral length is accurate for skeletons over six feet in height, but we cannot comment on this with certainty. Cadaver specimens in this collection do not have any remaining cartilage and hence the wear patterns often seen during TKRs could not be accounted for in our study. Systematic differences in the thickness of the cartilage or gender differences in the patterns of cartilage wear could not be assessed. The data for evaluation of the distal femoral cut of $8 \mathrm{~mm}$ was obtained from MATLAB calculations and simulation. No cuts were made on the specimens themselves.

This is a large anatomical study which shows that there is a difference in the aspect ratio between genders, but also that there is a high degree of variation between individuals. No difference between genders could be found for the heights of either the lateral or medial flanges. Future work in the design of knee prostheses should take into account the overall variability of the anatomy of the distal femur between individuals.

The authors would like to acknowledge Dr Haile-Selassie and L. Jellema for their work in the preparation of the manuscript. The views expressed in this article are those of the author(s) and do not necessarily reflect the official policy or position of the Department of the Navy, Department of Defence or the United States Government.

The Department of Orthopaedics at University Hospitals Case Medical Centre provided financial support for statistical analysis through the Resident Education Fund.

No benefits in any form have been received or will be received from a commercial party related directly or indirectly to the subject of this article.

\section{References}

1. Lonner JH, Jasko JG, Thomas BS. Anthropomorphic differences between the distal femora of men and women. Clin Orthop 2008;466:2724-9.

2. Emerson RH Jr, Martinez J. Men versus women: does size matter in total knee arthroplasty? Clin Orthop 2008;466:2706-10.

3. Clarke HD, Hentz JG. Restoration of femoral anatomy in TKA with unisex and gender-specific components. Clin Orthop 2008;466:2711-16.

4. MacDonald SJ, Charron KD, Bourne RB, et al. The John Insall Award: genderspecific total knee replacement: prospectively collected clinical outcomes. Clin Orthop 2008;466:2612-16.

5. Mahfouz MR, MerkI BC, Fatah EE, Booth R Jr, Argenson JN. Automatic methods for characterization of sexual dimorphism of adult femora: distal femur. Comput Methods Biomech Biomed Engin 2007;10:447-56.

6. Conley S, Rosenberg A, Crowninshield R. The female knee: anatomic variations J Am Acad Orthop Surg 2007;15(Suppl 1):31-6.

7. Kim YH, Choi Y, Kim JS. Comparison of standard and gender-specific posterior cruciate-retaining high-flexion total knee replacements: a prospective, randomised study. J Bone Joint Surg [Br] 2010;92-B:639-45.

8. Hitt K, Shurman JR 2nd, Greene K, et al. Anthropometric measurements of the human knee: correlation to the sizing of current knee arthroplasty systems. J Bone Joint Surg [Am] 2003;85-A:115-22.

9. Chin KR, Dalury DF, Zurakowski D, Scott RD. Intraoperative measurements of male and female distal femurs during primary total knee arthroplasty. J Knee Surg 2002;15:213-17. 
10. Fehring TK, Odum SM, Hughes J, Springer BD, Beaver WB Jr. Differences between the sexes in the anatomy of the anterior condyle of the knee. $J$ Bone Joint Surg [Am] 2009;91-A:2335-41.

11. Merchant AC, Arendt EA, Dye SF, et al. The female knee: anatomic variations and the female-specific total knee design. Clin Orthop 2008;466:3059-65.

12. Reichs KJ. Forensic osteology: advances in the identification of human remains National Criminal Justice (NCJ) 103038, 1998. http://www.ncjrs.gov/app/publications/abstract.aspx?!D=103038 (date last accessed 29 October 2010).
13. Bellemans J, Carpentier K, Vandenneucker H, Vanlauwe J, Victor J. The John Insall Award: both morphotype and gender influence the shape of the knee in patients undergoing TKA. Clin Orthop 2010;468:29-36.

14. Vaidya SV, Ranawat CS, Aroojis A, Laud NS. Anthropometric measurements to design total knee prostheses for the Indian population. J Arthroplasty 2000;15:79-85.

15. Cheng FB, Ji XF, Lai Y, et al. Three dimensional morphometry of the knee to design the total knee arthroplasty for Chinese population. Knee 2009;16:341-7.

16. McDowell MA, Fryar CD, Ogden CL, Flegal KM. Anthropometric reference data for children and adults: United States, 2003-2006. National Health Statistics Reports 2008;10. http://www.cdc.gov/nchs/data/nhsr/nhsr010.pdf (date last accessed 29 October 2010). 